\title{
Design and Autonomous Control of the Active Adaptive Suspension System of Rudra - Mars Rover
}

\author{
Karan Vaish ${ }^{*}$, Shah Mihir Rajesh ${ }^{* *}$, Karthikeyan Pasupatheeswaran, Manoj Kumar \\ Sharma and Vijay B. Semwal \\ SRM University, India \\ Siemens, India \\ *karan.vaish.93@gmail.com, ${ }^{* *}$ shahmihirrajesh@srmuniv.edu.in
}

\begin{abstract}
Semi or completely autonomous unmanned vehicles, remotely driven or controlled through artificial intelligence, are instrumental to foster space exploration. One of the mos essential tasks of a rover is terrain traversing which requires the need of efficient suspension systems. This communication presents a suspension system giving degrees" of freedom to every wheel with the help of linear actuators connected through bell gronklesers. The actuation of linear actuators directly varies the height of every wheel from the chassis hence ffering articulation to the rover. A control system is developed offering an algorithm for its autonomous actuation. This system proves instrumental for leveling of the chassis where any kind of slope, roll or pitch, may impute abstaining of payloads from efficient working. This was tried and tested successfully as a part of the rover developed by Team RUDRA from SRM University, INDIA (first Team from Asia and finishing at the fifth position at University Rover Challenge 2013, held at UTAH, USA in May-June.
\end{abstract}

Keywords: Bell Crank, Terrain traversing, Active Adaptive Suspension, Mars Rover.

\section{Introduction}

The use of automobiles has been augmented with every passing day. The automobiles remain unswerving, and move smoothly across various drive conditions with the help of a suspension. These suspen $10 n$ systems arexesponsive to the slightest of jerks on the course of mobility [1]. There are two main functions of a suspension system:

Primary function is to isolate the body from shocks and suspensions caused by the irregularities in the drive field; secondly, to establish constant contact between the wheels and the surface to provide good handling [2]. The suspension system consists of a spring and a damper, but this traditional setup cannot produce an active control force adaptive with the diversifying driving conditions.

Rover can be called as unmanned automobiles that are either autonomous or remotely controlied These rovers are used for many purposes among which space explorations are most widely implemented. In 2004, NASA sent two planetary rovers to Mars, called Sprit and Opportunity [3][4]. These rovers proved instrumental in answering numerous questions on extraterrestrial life. This successful mission proved the essence of rovers in space exploration.

While on celestial bodies, rovers need to traverse terrains that are heavily cratered or very smooth [5]. To maintain this much required stability on the system, a variety of suspension systems are incorporated like: Double Wishbone, Rocker Bogie, Strut Bar, the SHRIMP system 
[6], and PEGASUS system [7]. The Rocker Bogie used in the rover CURIOSITY has been NASA's most favored system.

Nevertheless, all the above-mentioned systems are passive suspension systems. In areas demanding high stability and travel ability, the active suspensions are the preferred choice [8]. However, these active suspension systems are slightly heavy, complex and consume more energy.

Most of the active suspensions concentrate on achieving greater traction coefficients and adaption to irregularities such as rocks. The presented suspension system, based on every single wheel being connected to a linear actuator through a bell crank lever, aims primarily at leveling the chassis where any kind of slope determined by a change in both pitch and roll axes may prove to be the biggest detriment to the smooth functioning of any onboard science payload. Such a system also offers increased traction capabilities and moment balancing for unbalanced loads. Incase, of loose soil etc., this system be used efficiently transfer loads, between different wheels for greater grip.

First we shall discuss the mechanical aspects highlighting various materials, key components if the design, the assembly and control over pitch and roll Further we shall move on to the electrical and the control design aspect of the system concentrating on the active adaptive control system.

\section{Key Elements Of The System}

\subsection{Bell Crank Lever}

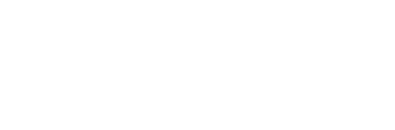

One of the most common mechanical linkages, a bell crank lever can simply be considered as a link that transfers force or a moment through a certain angle. This angle can vary from 0 to 360 degrees. But it usually calls for a tradeoff between range and linearity. Figure 1 depicts a simple bell crank lever setup illustrating the relationship between forces, the angular speeds and hence the basic kinematic relationships between the wo ends, experienced as a function of the arm length. Here, F1 and F2 depict the forces acting at each of the arms of lengths L1 and L2 respectively inclined at an angle of $\alpha$ to each other. The solid and the dashed lines demarcate the initial and final positions of the system, where both the links have respectively experience linear change of $\Delta \mathrm{x}$ and $\Delta \mathrm{y}$ respectively

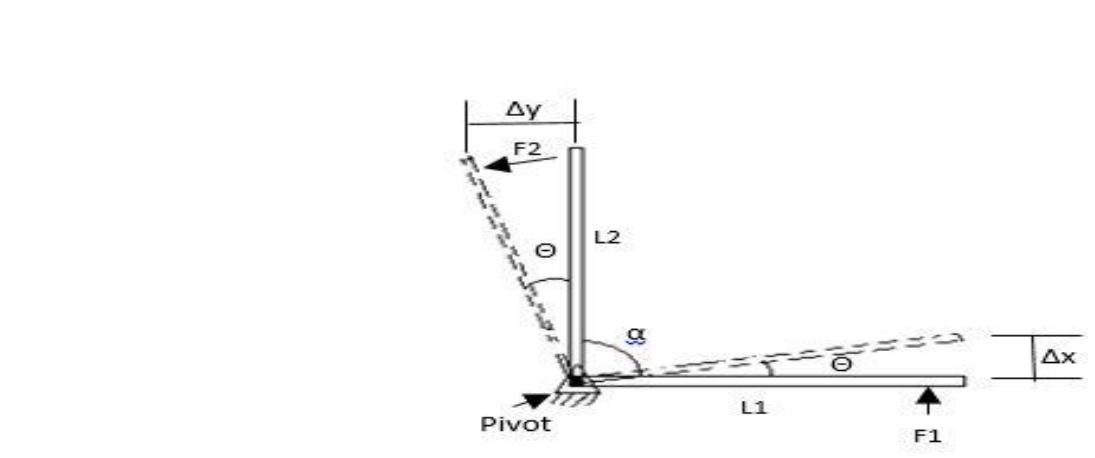

Figure 1. Bell Crank Lever

From concept of sum of net internal moments and forces being zero, we can say that

$$
F 1 \times L 1=F 2 \times L 2
$$




$$
\frac{F 1}{F 2}=\frac{L 2}{L 1}
$$

The same equations can be manipulated in order to encompass the linear changes between the initial and the final states of the system. Eq. (3), (4) and (5) determine the relationship between the changes in both the links.

$$
\begin{gathered}
\theta \times L 1=\Delta x \\
\theta \times L 2=\Delta y \\
\frac{\Delta x}{\Delta y}=\frac{L 1}{L 2}
\end{gathered}
$$

\subsection{Electric Linear Actuators}

Any electronic device that results in a change of state in terms of position of another object can be termed as an actuator. In other words it may be referred to as a control mechanism. Linear actuators are specifically those that deliver or create motion in a straight line. They can be driven by pneumatics, hydraulics or even electric motors manipulated through certain gear ratios. An often-realized design employs a simple motor and a serew configuration that displaces an attached nut with the apparent sliding tube. Fig. $\mathbf{2}$ depicts a simple schematic diagram of a commonly used electric linear actuator. The Electic Linear Actuator that was selected for use also contains a built in potentiometer, which gives a feedback to the controller and enables to sense the amount of extension and retraction.

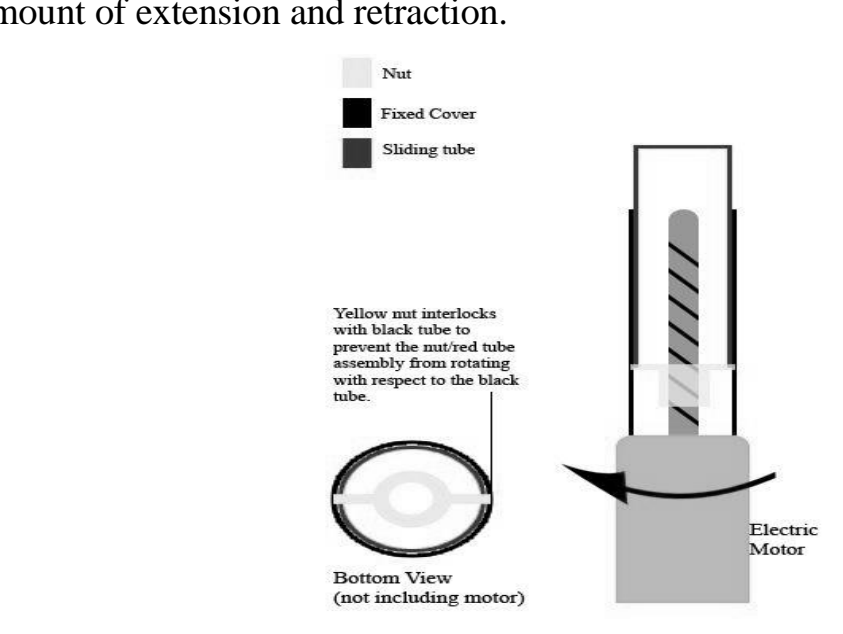

\section{Design And Assembly}

Figure 2. Schematic Diagram of an Electric Linear Actuator

The above-mentioned elements form the Primary components of the design such a chassis that can offer isolation and absorption of vibrations and also articulation to the chassis to match the profile of the terrain. Here each wheel is attached to the chassis through such a setup involving one linear actuator connected to the wheel via a bell crank lever. Fig. 3 shows the schematic side view of the whole setup. 


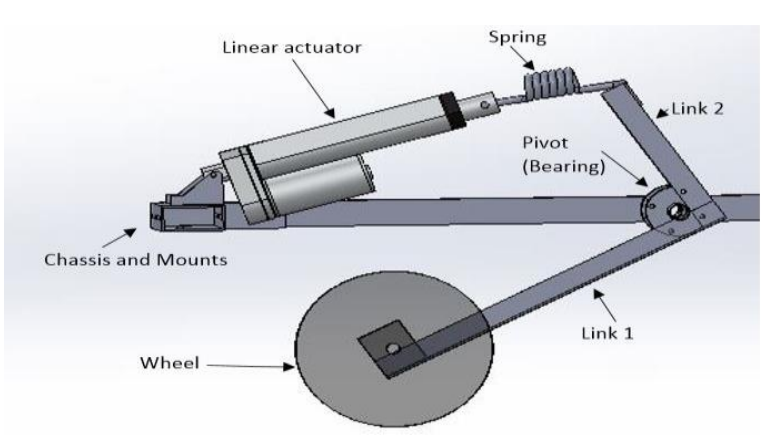

Figure 3. Arrangement of Components

It must be realized that this system purports independent articulation of each and every wheel and not just one single wheel with respect to the other. Depending on the gradient of the terrain the linear actuators can be manipulated to impute the much-required changes in the distance of the wheel centers (placed at the other end of the bell crank lever) to the botton of the chassis. These actuators are electronically controlled and the architecture is explained later in this communication.

Figure 4 and Eq. (6) to (14) highlight the relationship between the extension of the linear actuator required to obtain the required the change in height of that specific point on the chassis to the ground.

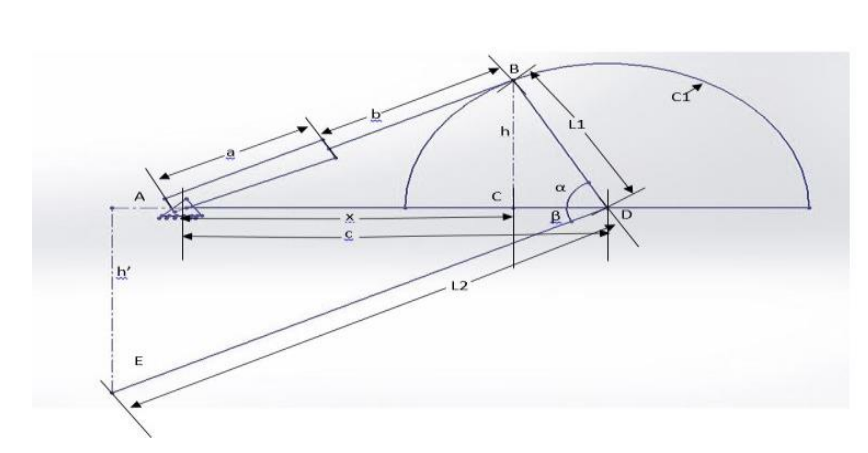

Figure 4. System Configuration

In order to empirically derive a relationship between the amount of actuation required to produce a desired change in the height, let us first consider triangle $\mathrm{ABC}$

$$
\text { (a+b) })^{2}=h^{2}+x^{2}
$$

Also from triangle $\mathrm{BCD}$

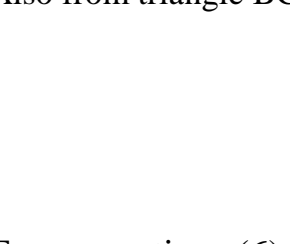

$$
\begin{gathered}
x=c-(L 1 \cos \alpha) \\
h=(L 1 \sin \alpha)
\end{gathered}
$$

From equations (6), (7) and (8), we can see that

$$
\begin{aligned}
& (a+b)^{2}=(L 1 \sin \alpha)^{2}+[c-(L 1 \cos \alpha)]^{2} \\
& b=\sqrt{(L 1 \sin \alpha)^{2}+[c-(L 1 \cos \alpha)]^{2}}-a .
\end{aligned}
$$


Now, considering the triangle ADE, to incorporate the Link 2 we see that,

$$
\begin{gathered}
\beta=90^{\circ}-\alpha, \\
h^{s}=L 2 \sin \left(90^{\circ}-\alpha\right) \\
\alpha=90^{\circ}-\sin ^{-1} \frac{h^{r}}{L 2} \quad \text { (13) }
\end{gathered}
$$

Substituting these equations back in equation (10) we get a relationship between the desired height and the amount of actuation required, i.e.:

$$
b=\sqrt{\left\{L 1 \sin \left(90^{\circ}-\sin ^{-1} \frac{h^{\prime}}{2}\right)\right\}^{2}+\left[c-\left\{L 1 \cos \left(90^{\circ}-\sin ^{-1} \frac{h^{\prime}}{2}\right)\right\}^{2}\right.}-a
$$

Table 1. Key

\begin{tabular}{|l|l|}
\hline Symbol/letter & Description \\
\hline $\mathrm{a}$ & Unactuated length \\
\hline $\mathrm{b}$ & Extension \\
\hline $\mathrm{c}$ & Distance between pivot or the actuator and Lever \\
\hline $\mathrm{x}$ & $\begin{array}{l}\text { Distance between the pivot of the actuator and the } \\
\text { perpendicular distance at the point of the contact }\end{array}$ \\
\hline $\mathrm{h}$ & Height from the chassis of the poin of contact \\
\hline $\mathrm{L} 1$ & Link 1 \\
\hline $\mathrm{L} 2$ & Link 2 \\
\hline$\alpha$ & Angle between Link 1 and chassis \\
\hline$\beta$ & Angle between link 2 and chassis \\
\hline $\mathrm{h}$ & Height of the chassis from the wheel center \\
\hline
\end{tabular}

Since, we are concerned with a drive consisting of four independently powered wheels, four of these mechanisms were coupled together to form a complete chassis. Figure 5 shows a model generated on Solidworks depicting clearly the arrangement and configurations of each and every crucial component of the 4-wheeled assembly.

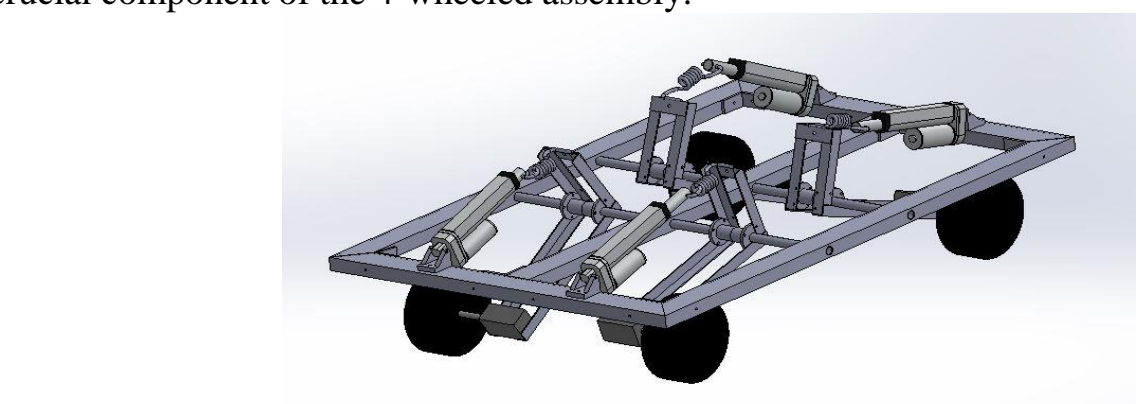

Figure 5. Complete Chassis

\section{Control of Roll And Pitch}

Roll, pitch and yaw are three main angles given by Trait-Bryan and Euler [16].

These set of angles correspond to the rotation of a rigid body across three perpendicular axes. Analogous to the $\mathrm{x}, \mathrm{y}$ and $\mathrm{z}$ axes, which could be considered as the roll, pitch or yaw axis. 
Since in our rover, the yaw is nothing but simple steering, it can be done by either differential or independent wheel steering mechanisms. In order not to digress from the main concern of the communication, it must be noted that the suspension aims in achieving optimal control in the roll and pitch axes, which are further discussed in the subsequent sections.

\section{A. Roll}

The roll axis here is being considered as the one that extends in the direction of forward motion of the rover. Respectively the front plane of the rover as marked in the Fig. 6 forms the actual plane of rotation. For the same, simple difference in lengths of the linear actuators of both front and rear of the left side wheels (W1, W2 respectively) as compared to the front and rear of the right side wheels (W4, W3) can impute to a change in roll angle. Greater the difference, greater is the roll angle. Fig. 6 shows the existence of roll angle due to a difference in the distance of the two sides, from the ground.

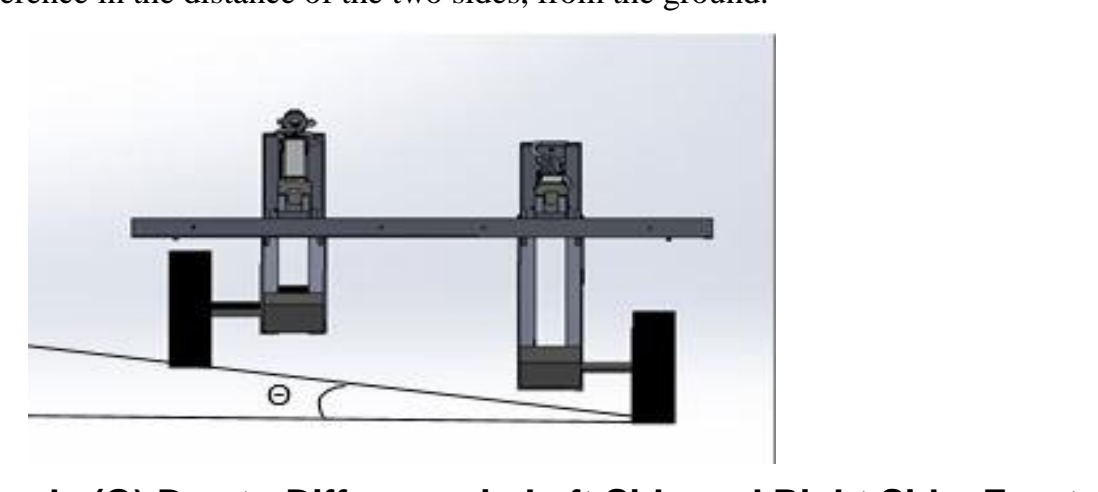

Figure 6. Roll Angle (๑) Due to Difference in Reft Side and Right Side; Front

\section{B. Pitch}

Pitch would eventually form the rotation about an axis perpendicular to both the roll and the yaw axes. Since both of these axes have been defined earlier in this communication, it must be understood that pitch axes would be the one experiencing rotation due to the difference of heights from the ground between the front two and the rear two wheels. Figure 7 depicts the pitching of the rover due to the difference in the front and rear end wheels.

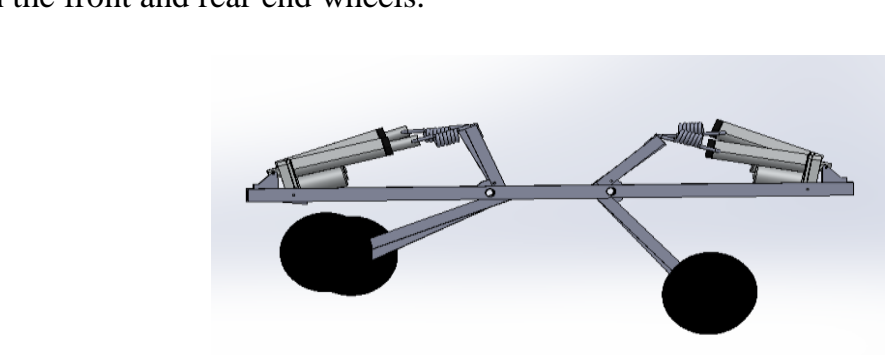

\section{Figure 7. Pitch Due to Difference in the Front and Rear Wheels; Side View}

Now, it is understood that during terrain traversing, one of the most primary and important tasks that any rover needs to perform in order for a successful mission, a rover has to experience a range of varying gradients. As mentioned earlier these can be either due to nominal irregularities in the ground profile or during ascent or descent of mountains or craters and valleys for rock and soil analysis. In either of the cases it is highly unlikely that either of the two rotations (roll or pitch) would occur at any given time. One may argue that a descent in a crater or a valley, like mentioned above, may impute only a change in the pitch angle for a rover, but it is not always that a rover traverses down such a patch straight. Often it spirals, and looks for 
the best possible path. In such a case, what the rover experiences is nothing but several combinations of the roll and pitch angles. In order to compensate for the roll and pitch angles one may simply calculate the resultant vector at any given time during the terrain traversing, as obtained, from the an inertial measurement unit and calculate the individual actuation lengths required from the linear actuators. But this kind of an approach would generally prove counterproductive due to an extremely slow response. Hence, by the time a certain configuration is achieved for incorporating the slope due to a certain part of the terrain, the terrain profile may have effectively changed hence rendering further instability to the system. For the same it is essential that a continuous process is realized which involves constant timely checks with the terrain. This mechanism is explained much in detail in the subsequent sections of this communication.

\section{Electronics Design}

The heart of Rudra-Mars Rover is the ATMega 2560, which permits communications and interfacing with the sensor peripherals, which are required for sensing parameters and transferring data to the Ground-Station. The 4 inch stroke length sophisticated/Electric Linear Actuators are controlled by Pololu Jrk USB Motor Controller by means of Pulse Width modulation technique. The advantage with the Concentric LD Series of Linear actuator is that it has a built in Potentiometer for feedback. One of the lasks in the URC was delivering the packages at given specified co-ordinates and thus Media-Tech GPS 3329 was used for it consumes less power and can be armed quickly while having the capability to access 66 satellites, is connected to the ATMega 2560 via Software Serial. The IMU, which is use to stabilization and control of the active adaptve suspension, is connected via Software Serial. Fig. 10 shows the CAD design of the IMU in which the outputs of all sensors are processed by an on-board ATmega328 and output over a serial interface [17]. This enables the IMU to be used as a very powerful control mechanism.

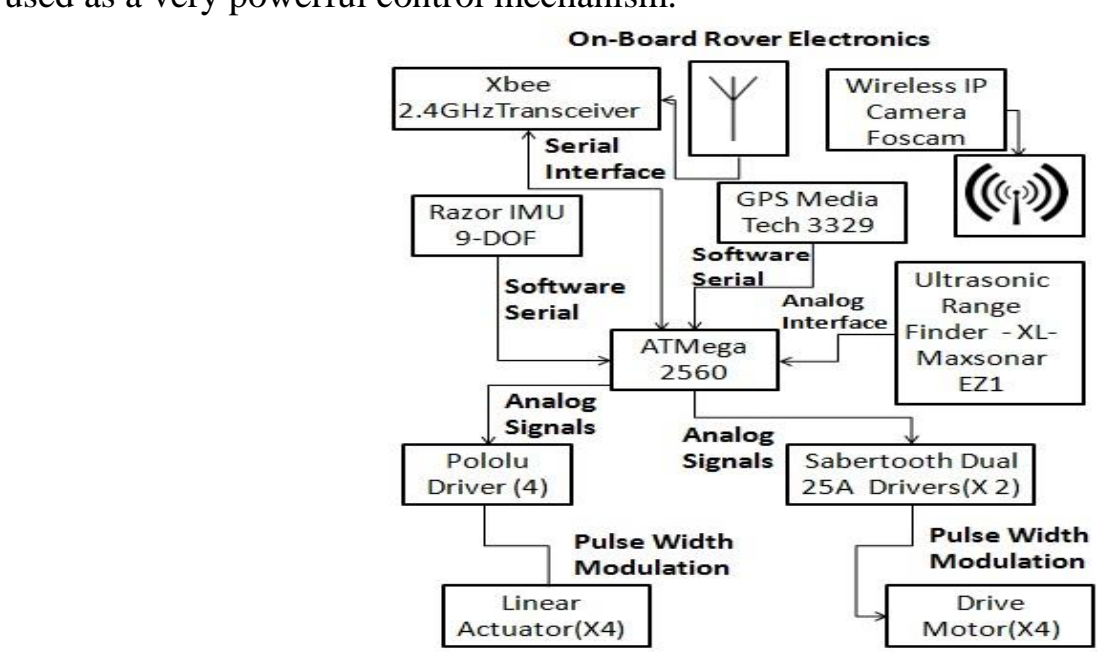

\section{Figure 8. Block Diagram Representation of On-Board Rover Electronics and Means of Communication with Controller}

Figure 8 explains the various peripherals communicating with the controller to send or receive data .The Drive of the Rover is controlled means of Sabertooth Drivers2X25 V2 and using the analogue mode for sending Pulse Width Modulation to the Drive Motors. The data from IMU, GPS and Ultrasonic is send via Xbee connected serially to the ATmega 2560 and the data is received in the Ground -Stating Docking as described by a block diagram representation in Fig. 9. The On-Board Wireless IP Camera is 
used for manoeuvre and terrain traversing of the Rover and is link with a Wi-Fi Modem setup at the Ground-Station Docking.

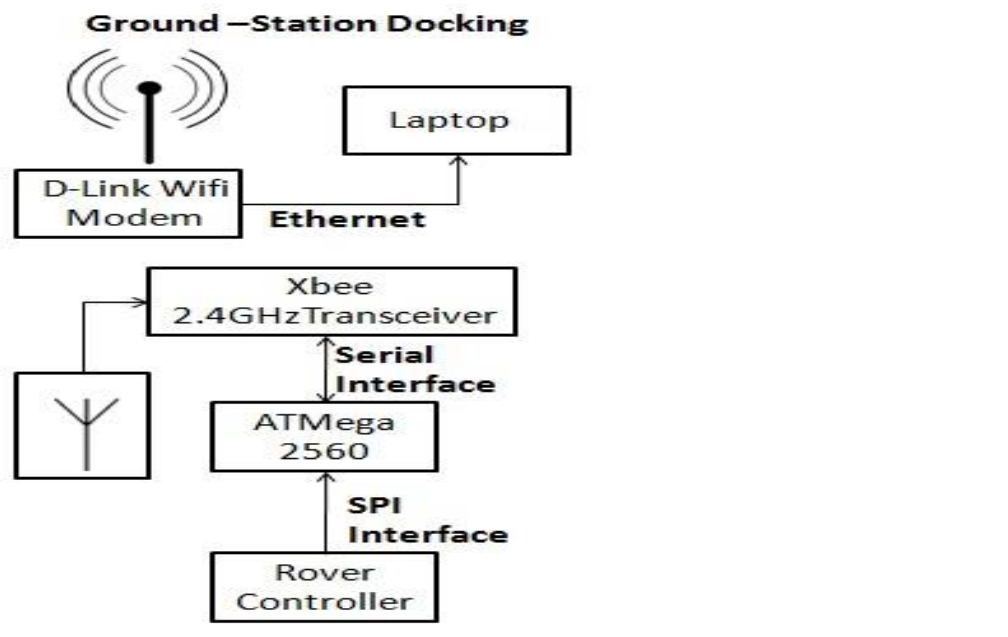

Figure 9. Block Diagram Representation of Ground Station Docking

The telemetry of data of IMU, Range sensor and GPS is done by means of Xbee at $2.4 \mathrm{GHz}$ and can be seen in the GUI setup to monitor the receiving data. The camera can be titled from the Docking station itself and is done via common inne 1 address of the camera configured with that of Wifi Modem.

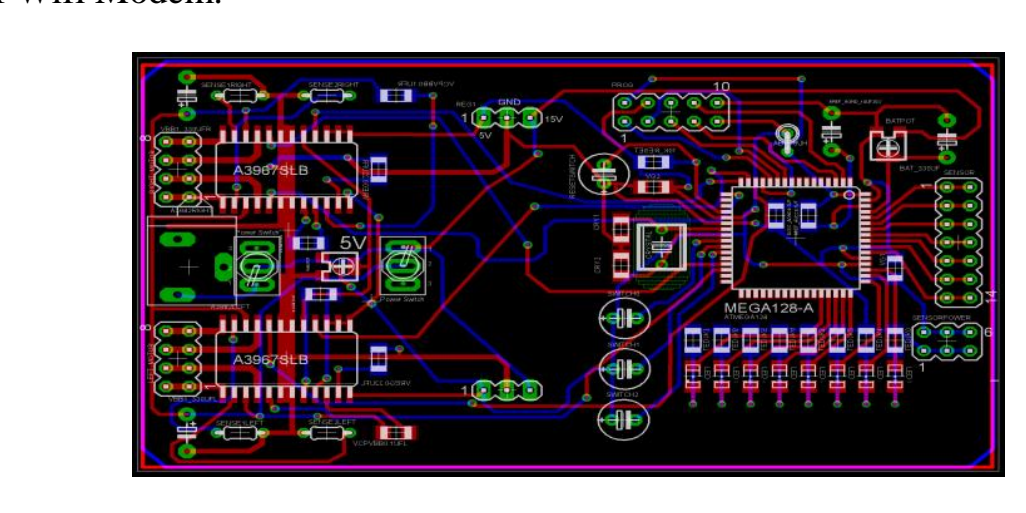

Figure 10. CAD Design of IMU Made using EAGLE [17]

\section{Electrical Design}

The Power distribution plays an important role as the Rover needs to traverse though the Desent for each task about 50 minutes. Table II shows the power budget sheet calculation, whick helps us to estimate the run-time of the Rover. Three 4S 6000mAh and 25C Turnigy Nano-Tech Batteries were used as the main Supply source and are connected in Series and hence the total run time is calculated to be roughly 72 minutes which is sufficient for each task. Fig. 11 shows the voltage distribution to each peripheral from the main supply. IC 7812 is used to supply power to each actuator as well as a heat sink is installed to reduce the temperature of the IC. LD1117V33 is used to convert to $3.3 \mathrm{~V}$ from $12 \mathrm{~V}$ with maximum current output capacity being one ampere. 


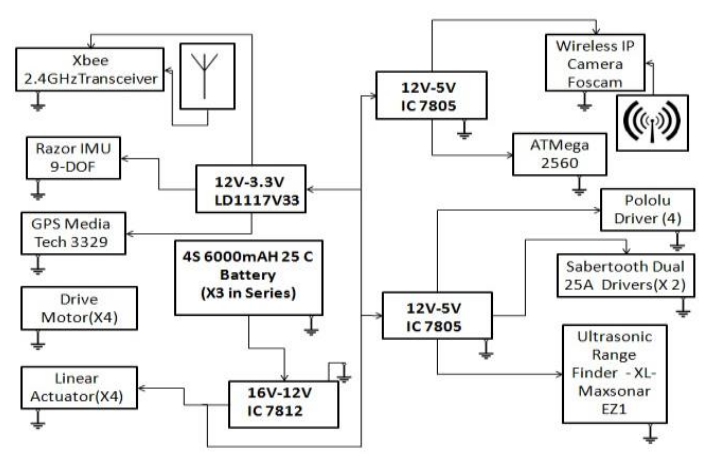

\section{Figure 11. Power Supply Distribution of the Rover}

Table II. Power Budget Sheet

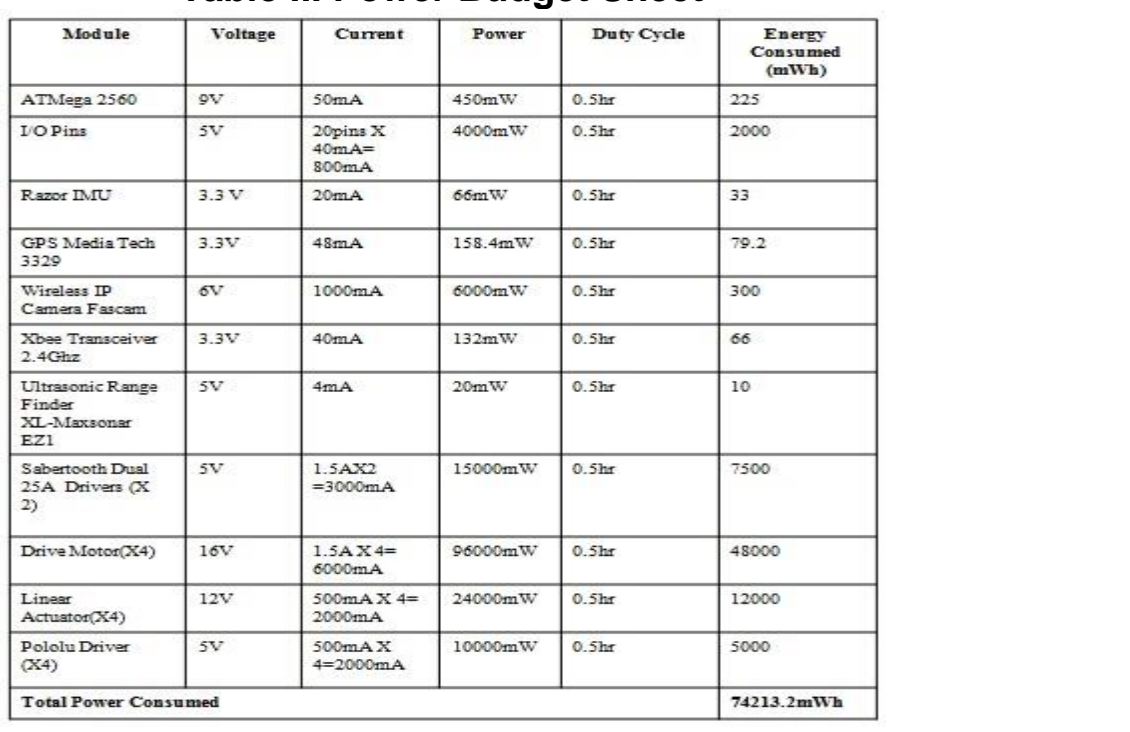

\section{Adaptive Control Design of Rudra}

The Key Elements of the Control System for Rudra include the IMU, Ultrasonic Range Finder and the Potentiometer Feedback from the Linear Actuators and the Actuators itself. The overall aim of the Confrol System designed is to maintain a Horizontal position of the Chassis autonomously by recovernng from each individual pitch and roll angles and maintain a constant or variable ground clearance depending upon the obstacle [9-13].

Figure 12 Explains about the Control System designed for Rudra. The ATmega 2560 senses from the IMU readings of ADXL345 and ITG-3200. The values obtained are ADC values and do not indicate an angle or acceleration. Eq. (15) - (17) shows the method of converting the IMO values to radians [14].

Thus we have used the following equation to convert it into respective angles:

$$
\text { GyroRate }=(\text { GyroAdc }- \text { GyroZero }) / \text { Sensitivity }
$$

The result of the above equation will be in as degrees per second. To convert this into degrees we need to multiply it by change in time, which is done by a function called millis (). Thus the final equation will be: 
The gyro cannot be used over a long duration due to drift and thus it becomes completely unreliable to trust on gyroscope completely. As seen above to measure the Gyro Angle, the same principle is used to measure the acceleration:

$$
\text { accVal }=\text { accAdc }- \text { accZero }
$$

The zero voltage at $0 \mathrm{~g}$ is approximately $1.5 \mathrm{~V}$ as seen in the datasheet of ADXL345 [18], to translate this into quids, we have used the following formula: zeroVoltage/3.3*1023.

$$
\begin{aligned}
& \text { Pitch }=\operatorname{atan} 2(\operatorname{acc} Y v a l, \text { accZval })+P I \\
& \text { Roll }=\operatorname{atan} 2(\operatorname{accXval}, \text { accZval })+P I
\end{aligned}
$$

For Pitch angle from Eq. (18) is the angle in radians between the positive Zaxis of the plane and the point given by the co-ordinates (accYval, accZval). The ange is positive for counter-clockwise angles (upper half-planeaccYval> 0), and negative for clockwise angles (lower half-planeaccYval < 0).Similarly the Roll angle can be measured.

Thus an accelerometer is used but it is unstable for a short span. As a result a combination is preferred to estimate the change in angle across each of the 3 axes by means of Kalman Filter. The IMU plays a very vital role in the active adaptation and hence the values were filtered. Eq. (20) - (26) are the common equations used to firter the yapues [15].

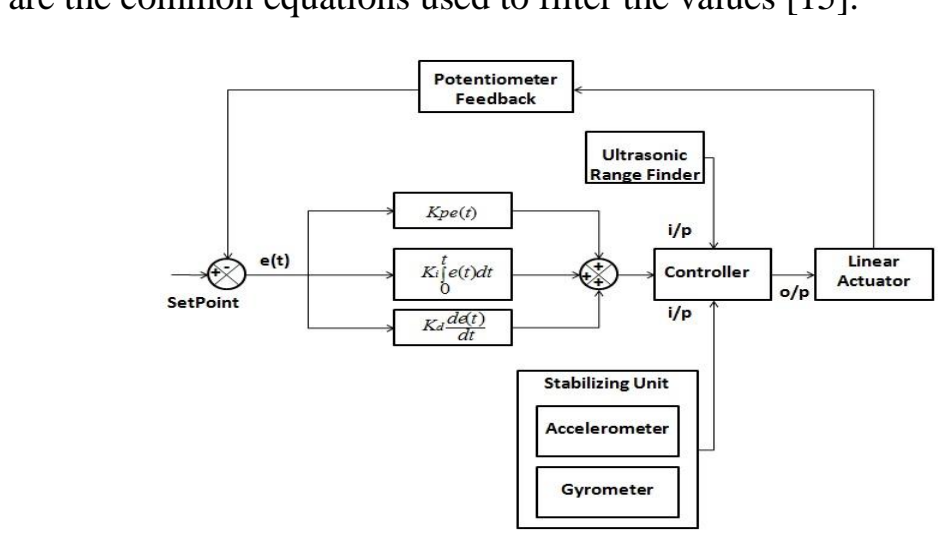

\section{Figure 12. Control Loop of the Active Adaptive System}

Eq.(20)predicts the current state by predicting the previous states and the Gyro measurement it is also called as control input, since it an extra input to estimate the state at the current time k .Eq. (21) explains about the error covariance matrix and it depends on the previous error covariance matrix as well as seen in the equation. Hence the greater the value of the error covariance the more unreliable is the current value of the estimated states [23-28].

$$
\begin{aligned}
& \hat{\boldsymbol{x}}_{k \mid k-1}=\boldsymbol{F} \hat{x}_{k-1 \mid k-1}+\boldsymbol{B} \dot{\theta}_{k} \\
& \boldsymbol{P}_{k \mid k-1}=\boldsymbol{F} \boldsymbol{P}_{k-1 \mid k-1} \boldsymbol{F}^{T}+\boldsymbol{Q}_{k}
\end{aligned}
$$


Eq.(22) is used to find the difference between the measurement and result obtained from Eq. (20).

$$
\tilde{y}_{k}=z_{k}-H \hat{x}_{k \mid k-1}
$$

The result of the Eq.(22)is also called as innovation. The observation model $\mathbf{H}$ is used to map the priori state into the observed space which is the measurement from the accelerometer. Eq. (23) shows the innovation covariance and its importance is that it tries to predict the reliability on trusting the measurement based on the a priori error covariance matrix obtained from Eq. (21)and the measurement covariance matrix $\mathbf{R}$. The observation model $\mathbf{H}$ is used to map the a priori error covariance matrix into observed space [29-34].

$$
\boldsymbol{S}_{k}=\boldsymbol{H} \boldsymbol{P}_{k \mid k-1} H^{T}+\boldsymbol{R}
$$

Eq.(24) gives the result of the Kalman gain and it states the reliability on the innovation obtained from Eq. (22). The Kalman Gain is used to predict the posterion of the current state and the posteriori covariance matrix is obtained from Eq. (26).

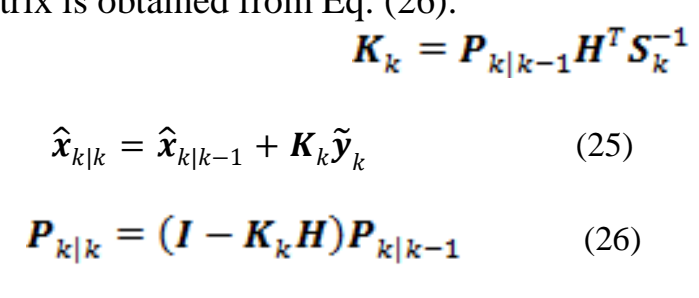

The Ultrasonic Range Sensor XL-Maxsonar EZlis placed at the center of the Rover along with the IMU. Now if there is an uneven terrain or the Rover is traversing along an uphill or a downhill, the IMU sends the Pitch and the Roli data to the Controller by means of Software Serial. Also the Range sensor calculates the distance of the Rover from the Ground-Station to predict the overall height in order to avoid obstacles [17-22]. Figure 13 shows the complete integration of electronics with the mechanical system.

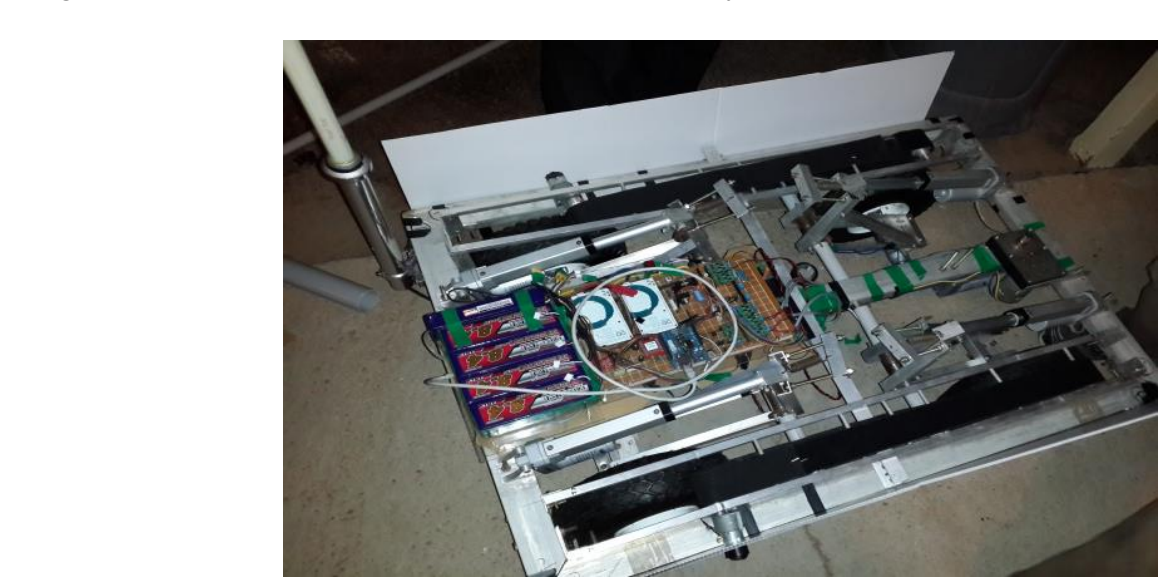

Figure 13. Electronics Setup on the Rover for Initial Testing

As mentioned earlier the overall goal of the control system, which is designed for Rudra, is to maintain and keep the chassis in a horizontal direction. This is done to avoid any fall of Payload as well as to overcome the slip. As soon as the controller is aware about the change in angle either a Pitch or Roll or even a combination of both, it tries to bring the values to zero and 
maintain stability. The output data from the controller to the linear actuator is mapped with the values of the IMU and is preloaded in the RAM. Another feedback is obtained from the linear actuator, which has an inbuilt potentiometer. The values of each potentiometer are mapped with that of the set point decided by the Range sensor and the IMU. For Example: If the Rover is at a Pitch angle of 20 degrees and a Roll of 40 degrees, then the Rover will first try to resolve the Roll angle as its effect on instability is maximum and then try to resolve the Pitch. The Range sensor is placed in the centre to have a greater degree of movement of flexibility for Roll and Pitch Recovery. There are 4 Linear Actuators, which control the movement of each wheel [18-19]. In order to stabilize the pitch angle either the front two or the rear two linear actuators are controlled. And to stabilize the Roll angle either the right or the left sided linear actuators are controlled. Figure 14 explains the Control Algorithm in the form of Flow Chart Diagram.

\section{Results}

Figure 14. Flow Chart Diagram of the Control Algorithm

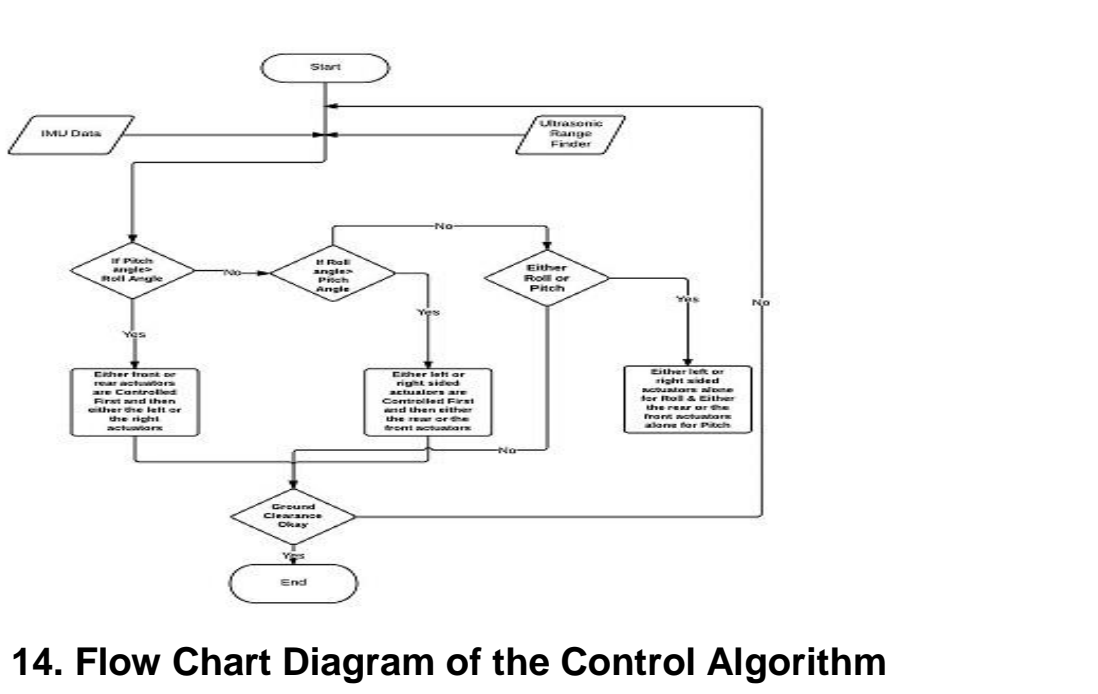

In order to check the response of the linear actuators when subjected to heavier payloads, Load v/s Speed graphs were plotted to check the compliance and also the feasibility before the Rover was made to run. Figue 14 shows the graph plotted and successful results were obtained as seen from the graph.

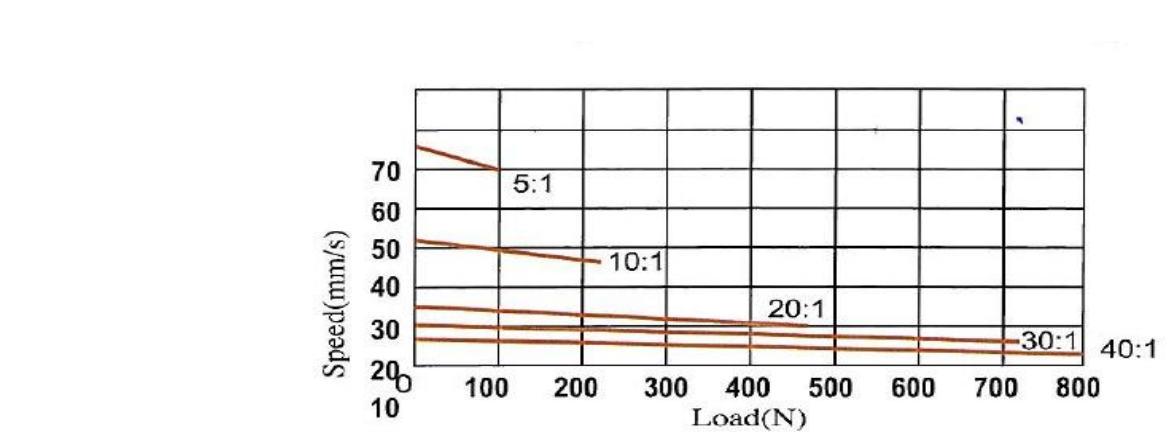

Figure 16. Graph Illustrating the Relationship between Load vs Speed Characteristics of the Linear Actuator

Further, the whole system was realized a month if April 2013 and was put through rigorous testing before the competition. Figure 16 shows the initial testing of the suspension system after 
incorporating the whole assembly. Figure 17 and Figure 18 show its real time operation in the Moab Desert in Utah, at the Mars Desert Research Station (MDRS) in USA.

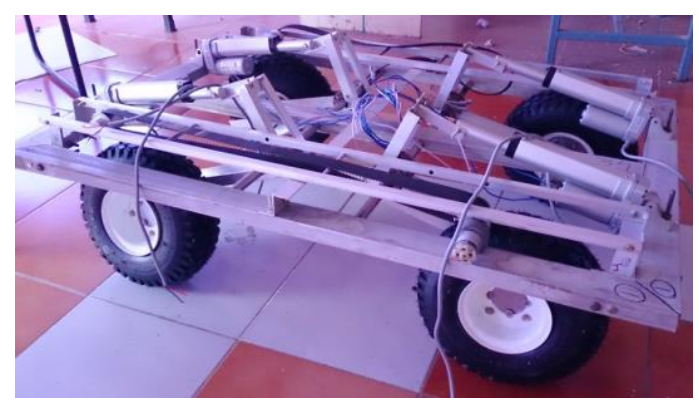

Figure 15. Setting up of Suspension System and Testing

Figure 17. Images of the Rover During Terrain Traversing and other Tasks
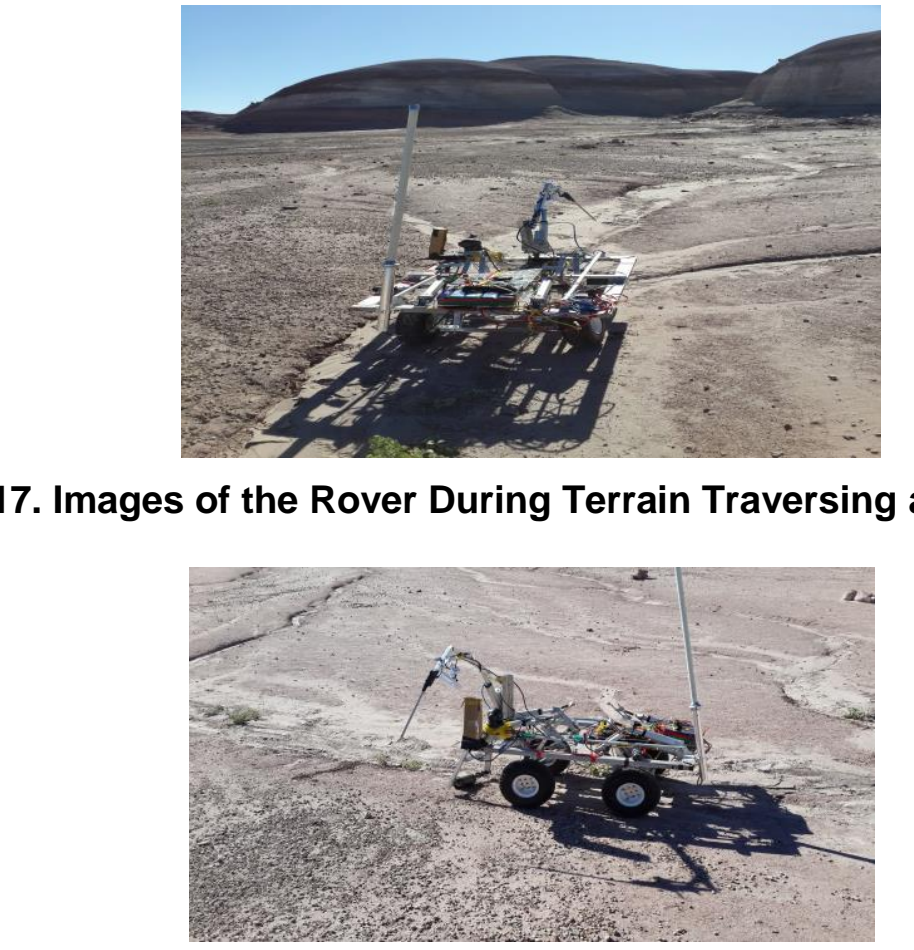

Figure 18. Images of the Rover During Terrain Traversing and other Tasks

\section{Conclusions}

his communication presented the mechanical and electrical design of a novel suspension system that purports individual degrees of freedom to every single wheel thus enhancing terram-traversing abilities of the rover. Through this communication first, the design followed by the assembly and complete control system was developed and discussed. The suspension proved instrumental in smooth traversing through the rough terrain rich with varying gradients and slopes. The chassis was found to be mostly horizontal unless the slope crossed the range of correction that the linear actuators could offer. It was understood that for greater angles, other linear actuators of higher stroke lengths should be adopted. 


\section{Acknowledgement}

The authors would like to acknowledge the efforts put in by Alekhya Gumidelli, R.B Jagadeesh, Rakesh Vasireddy, G.K Naren, Akshat Agarwal and Mohit Agarwal towards the completion and a successful performance at the competition.

Further, we would like to extend our gratitude to SRM University for its unwavering support in terms lab facilities and monetary resources for funding our research and participation.

\section{References}

[1] V. B. Semwal, K. S. Kumar, V. S. Bhaskar and M. Sati, “Accurate location estimation of moving object with energy constraint \& adaptive update algorithms to save data", arXiv preprint arXiv:1108.1321, (2011).

[2] K. S. Kumar, V. B. Semwal and R. C. Tripathi, "Real time face recognition using adaboost improved fast PCA algorithm”, arXiv preprint arXiv:1108.1353, (2011).

[3] K. S. Kumar, S. Prasad, S. Banwral and V. B. Semwal, "Sports Video Summarization using Priority Curve Algorithm", International Journal, vol. 2, (2010).

[4] K. S. Kumar, V. B. Semwal, S. Prasad and R. C. Tripathi, "Generating 3D Model Using 2D Images of an Object", International Journal of Engineering Science, (2011).

[5] V. B. Semwal, K. S. Kumar, V. S. Bhaskar and M. Sati, "Accurate locatron estimation of nioving object with energy constraint \& adaptive update algorithms to save data," arXiv preprint arXiv:1108.1321, (2011).

[6] J. P. Gupta, N. Singh, P. Dixit, V. B. Semwal and S. R. Dubey, "Human Activity Recognition using Gait Pattern", International Journal of Computer Vision and Image Processing, vol. 3, no. 3, (2013), pp. 31-53.

[7] V. Vikash, V. B. Semwal and P. Kumari, "A fault-tolerant mobile computing model based on scalable replica", International Journal of Interactive Multimedia and Arificial Intelligence (IJIMAI), (2014).

[8] S. R. Dubey, P. Dixit, N. Singh and J. P. Gupta Anfected Fruit Part Detection using K-Means Clustering Segmentation Technique", International Journâl of Interactive Multimedia and Artificial Intelligence, vol. 2, (2013), pp. 65-72.

[9] S. R. Dubey and A. S. Jalal, "Detection and Classification of Apple Fruit Diseases Using Complete Local Binary Patterns", In the Proceedings of the Third International Conference on Computer and Communication Technology, (2012), pp. $346-351$.

[10] N. Singh, S. R. Dubey, P. Dixit and J. P. Gupta, "Semant Image Retrieval by Combining Color, Texture and Shape Features", In the Proceedings of the International Conference on Computing Sciences, (2012), pp. $116-120$.

[11] S. R. Dubey and A. S. Jalal Adapted Approach for Fruit Disease Identification using Images", International Journal of Computer Vision and Image Processing, vol. 2, no. 3, (2012), pp. 44-58.

[12] S. R. Dubey and A. S. Jalal, "Species and variety detection of fruits and vegetables from images", International Journal of Applied Pattern Recognition vol. 1, no. 1, (2013), pp. 108-126.

[13] S. R. Dube and A. S. Jalal, "Robust Approach for Fruit and Vegetable Classification”, Procedia Engineering, vol. 38, (2012), pp. 3449-3453

[14] V. Bijalwan, V. Kumar, P. Kumari and J. Pascual, "KNN based Machine Learning Approach for Text and Document Mining", Intemational Journal of Database Theory \& Application, vol. 7, no. 1, (2014).

[15] Darus, R.; Enzai Nu Cldawati, "Modeling and control active suspension system for a quarter car model," Science and Social Research (CSSR), 2010 International Conference on Computer Applications and Industrial Electronics, vol., no., pp.1203, 1206, 5-7 Dec. 2010W.-K. Chen, Linear Networks and Systems (Book style) Belmont, CA: Wadsworth, 1993, pp. 123-135.

[16] LIU Shao-Jun, HUANG Zhang-hua, CHEN YI-Zhang, Automobile Active Suspension System with Fuzzy Logic College of Mechanical and Electrical Engineering, Central South University, Changsha 410083, China.

[17] Ha rington D. B., Voorhees J. C., The Challenges of Designing the Rocker-Bogie Suspension for the Mars Exploration Rover, Proc.ofthe37thAerospace Mechanisms Symposium (2004), NASA/CP-2004- 212073.

[18] Seeni A., Schafer B., Rebele B., Tolyarenko Niko- lai., Robot Mobility Concepts for Extraterrestrial

[19] Hershenson, M. (Ed.) (1989). The Moon Illusion. Lawrence Erlbaum \& Associates, Hillsdale,NJ

[20] Michaud, S., Schneider, A., Bertrand, R., Lamon, P., Siegwart, R., van Winnendael, M., Schiele, A., SOLERO : Solar Powered Exploration Rover, Proc. of the 7 ESA Workshop on Advanced Space TechnologiesforRoboticsandAutomation(2002).

[21] KurodaY., KondoK., NakamuraK., KuniiY., Kub- ota T., Low Power Mobility System for Micro Plan- etary Rover Micro5, Proc. of the 5th International Symposium on Artificial Intelligence, Robotics and AutomationinSpace(1999),pp.77-82. 
[22] Huntsberger, T. L., Baumgartner, E. T., Aghazarian, H., Cheng, Y., Schenker, P. S., Leger, P. C., Iag- nemma, K. D., and Dubowsky, Sensor-Fused Autonomous Guidance of a mobile robot and applica- tions to mars sample return operations, Proc. SPIE Int.SymposiumonIntelligentSystemsandAdvanced Manufacturing,vol.3839(1999),pp.2-8.

[23] The Evaluation of Planetary Rover for Rough Terrain by Takamasa Naiki and Takashi Kubota

[24] Gupta, Jay Prakash, Nishant Singh, Pushkar Dixit, Vijay Bhaskar Semwal, and Shiv Ram Dubey. "Human Activity Recognition using Gait Pattern." International Journal of Computer Vision and Image Processing (IJCVIP) 3, no. 3 (2013): 31-53.

[25] Matt Bird,Tina Sandaval,Matt Wicke,Mark Nenninger,Self -Orientating Autonomous Pathfinder,2011 NASA Research Grant.

[26] Karl Iagnemma, Robert Burn, Eric Wilhelm,Steven Dubowsky,Experimental Validation of Physics-Based Planning and Control Algorithms for Planetary Robotic Rovers,International Symposium on Experimental Robotics 1999.

[27] J.Pijuan,M.Comellas,M.Nogues,J.Roca,X.Potau,Active bogies and chassis levelling for vehicle operating in rough terrain,Journal of Terramechanics, Volume 49,Issue 3-4,June -August 2012,Pages 161-171

[28] Mahmoud Tarokh,Huy Dang Ho,Antonio Bouloubasis,Systematic kinematics analysis and balance of high mobility rovers over rough terrain, Elsevier Robotics and Autonomous Systems, Volume 61, Issue 1 ,January 2013,Pages 13-24.

[29] https://www.sparkfun.com/products/10736.

[30] http://forum.arduino.cc/index.php?topic=58048.0.

[31] http://blog.tkjelectronics.dk/2012/09/a-practical-approach-to-kalnran-filter-and-how-to-inplement-it/

[32] Novi Commentarii academiae scientiarum Petropolitanae 20, 1776, pp. 189-207 (E478).

[33] Espada, Jordán Pascual, Rubén González Crespo, Oscar Sanjuán Martínez, G. Cristina Pelayo, B. Bustelo, and Juan Manuel Cueva Lovelle. "Extensible architecture for context-aware mobile web applications." Expert Systems with Applications 39, no. 10 (2012): 9686-9694.

[34] Crespo, Rubén González, Sergio Ríos Aguilar, Roberto Ferro Éscobar, and Nicolás Torres. "Dynamic, ecological, accessible and 3D Virtual Worlds-b̂ased Libraries using OpenSim and Sloodle along with mobile location and NFC for checking in." International Journal of Interaetive Multimedia \& Artificial Intelligence 1, no. 7 (2012).

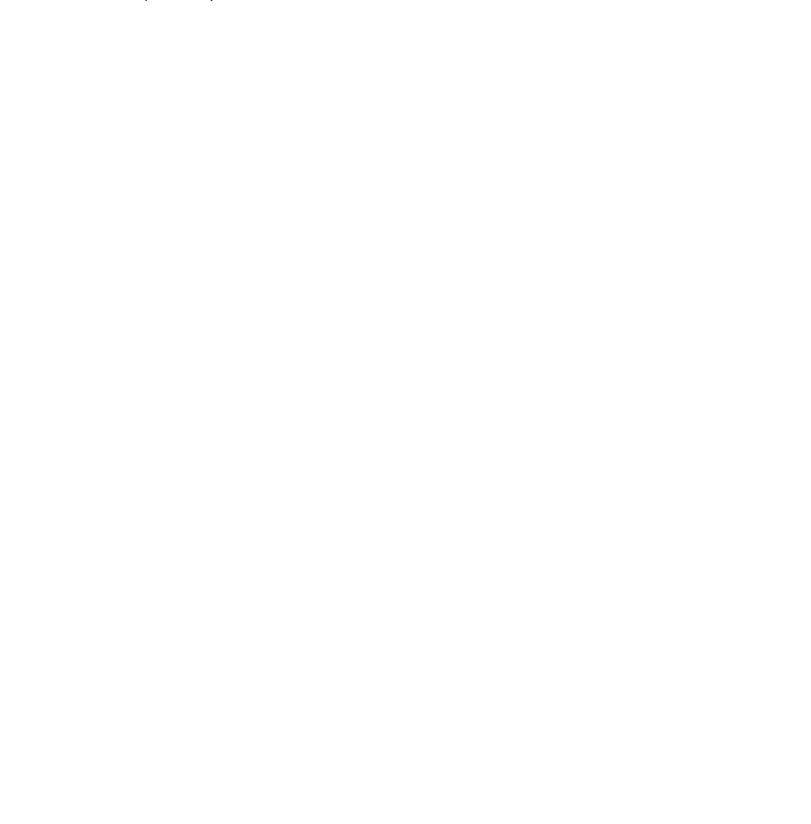


International Journal of Hybrid Information Technology

Vol.7, No.3 (2014)

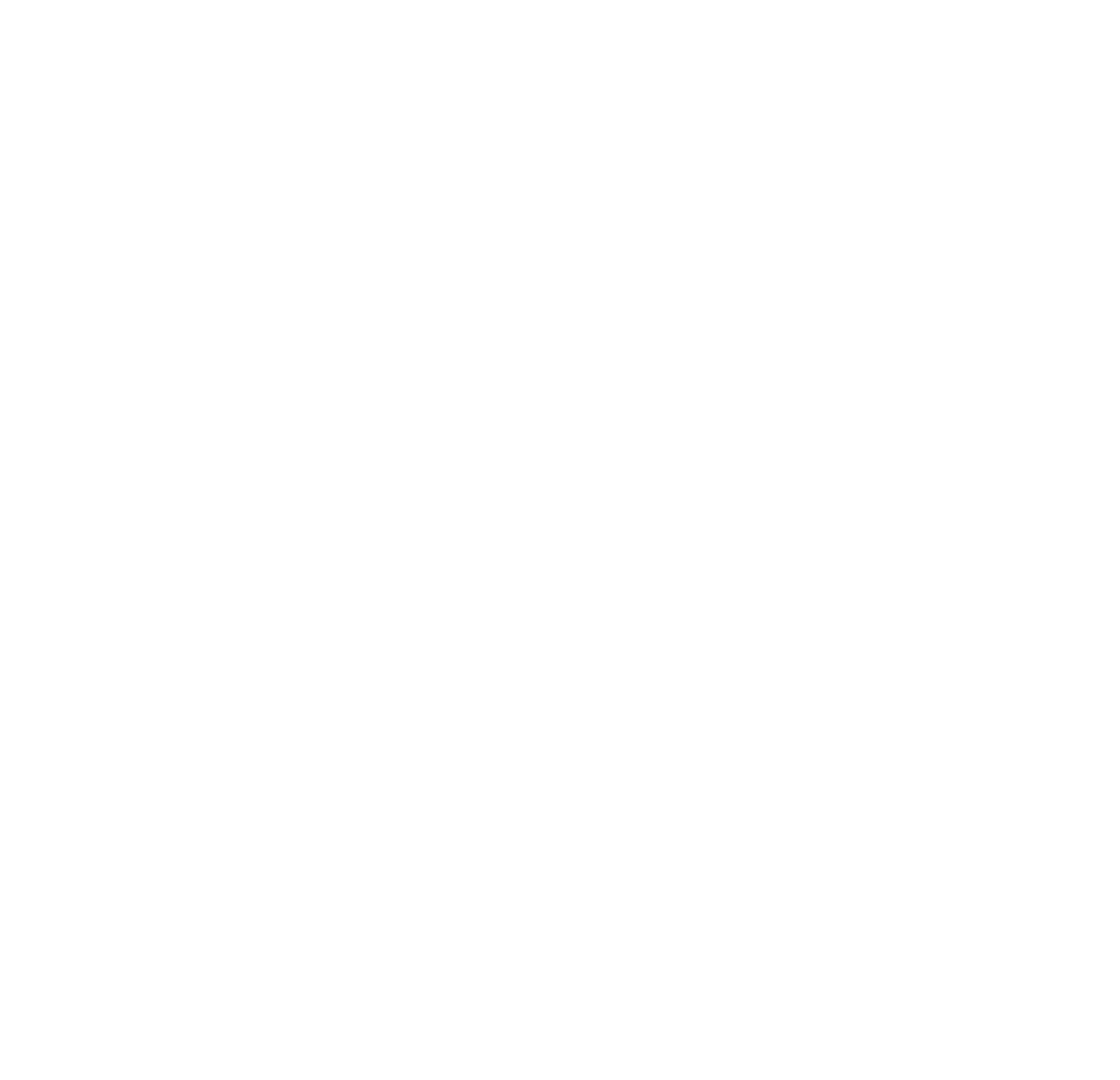

